\title{
Clinical Presentation of Gastrointestinal Stromal Tumors
}

\author{
Franka Menge $^{a} \quad J^{2} n s J_{a k o b}{ }^{b} \quad$ Bernd Kasper $^{c} \quad$ Armand Smakic $^{d}$ Timo Gaiser $^{\mathrm{e}}$ \\ Peter Hohenberger ${ }^{\mathrm{a}}$
}

\begin{abstract}
${ }^{a}$ Division of Surgical Oncology, Mannheim University Medical Center, Medical Faculty Mannheim, University of Heidelberg, Mannheim, Germany;

${ }^{b}$ Department of Surgery, Mannheim University Medical Center, Medical Faculty Mannheim, University of Heidelberg, Mannheim, Germany; ' Interdisciplinary Tumor Center, Mannheim University Medical Center, Medical Faculty Mannheim, University of Heidelberg, Mannheim, Germany;

d Institute of Clinical Radiology and Nuclear Medicine, Mannheim University Medical Center, Medical Faculty Mannheim, University of Heidelberg, Mannheim, Germany,

e Institute of Pathology, Mannheim University Medical Center, Medical Faculty Mannheim, University of Heidelberg, Mannheim, Germany
\end{abstract}

\section{Keywords}

Gastrointestinal stromal tumors, GIST · Clinical presentation

\section{Summary}

Gastrointestinal stromal tumors (GISTs) are the most common mesenchymal neoplasms of the gastrointestinal tract. They constitute $1-2 \%$ of all gastrointestinal neoplasms but are the most common subtype of soft tissue sarcomas, accounting for $20-25 \%$. In the late 1990s, GISTs were more and more recognized as a particular tumor entity. The tumors are supposed to originate from the interstitial pacemaker cells of Cajal. They are usually well circumscribed and can be located in every part of the tubular gastrointestinal tract. Most often GISTs occur in the stomach, followed by the small bowel and colon/ rectum. In contrast to epithelial tumors, GISTs grow transmurally and submucosal. GISTs can be found with highly variable growth features including tumors with intraluminal, intra- or transmural, and pedunculated appearance. Here we describe the most common clinical presentation of GISTs on the basis of our 809 patients managed from 2004 to 2017. The median age of our patients was 59 years and the average size of GIST was 75 $\mathrm{mm}$ (range: $4 \mathrm{~mm}$ to $35 \mathrm{~cm}$ ). The clinical presentation is very heterogeneous, depending on tumor site, size, and growth pattern. GISTs of the stomach is the group with the lowest rate of acute or emergency symptoms with $31 \%$, followed by GISTs of the duodenum with $42 \%$, whereas GISTs of the small bowel show acute symptoms in more than $50 \%$ of the cases and have an emergency surgery rate of almost 15\%. Many patients are diagnosed accidentally, through screening examinations, or with latent, unspecific symptoms.

(c) 2018 S. Karger GmbH, Freiburg

\section{Introduction}

Sarcomas are a heterogenic group of malignancies. Their cumulative incidence is about $1-2$ per 100,000 per year. $20-25 \%$ of these tumors originate from the gastrointestinal tract. The most common mesenchymal (non-epithelial) neoplasm of the gastrointestinal tract are gastrointestinal stromal tumors (GISTs) with about 10 cases per million inhabitants/year in Europe. In 1988, Hirota et al. [1] reported the activating KIT mutation and laid the basis that GIST could be defined as a particular tumor entity. GISTs are typically well circumscribed tumors that can be located in all parts of the tubular gastrointestinal tract. Very rarely, GISTs might originate from other intra-abdominal tissue. Their cellular origin is proposed to be the intestinal cells of Cajal - or a shared common stem cell. The cells of Cajal are intestinal pacemaker cells located between the layers of the muscularis propria, having immunophenotypic and ultrastructural features of smooth muscle and neuronal differentiation. The relationship of KIT expression and intestinal motility has been described long ago, but the exact relationship and the development of GIST remain unclear [2].

\section{KARGER}

(๑) 2018 S. Karger GmbH, Freiburg

Fax +497614520714
Dr. med. Franka Menge

Division of Surgical Oncology, Mannheim University Medical Cente

Medical Faculty Mannheim, University of Heidelberg

Theodor-Kutzer-Ufer 1-3, 68167 Mannheim, Germany

Franka.Menge@umm.de 
Fig. 1. Example of a patient with Carney triad with a pulmonary chondroma and $\mathbf{b}$ gastrointestinal stromal tumor of the stomach.
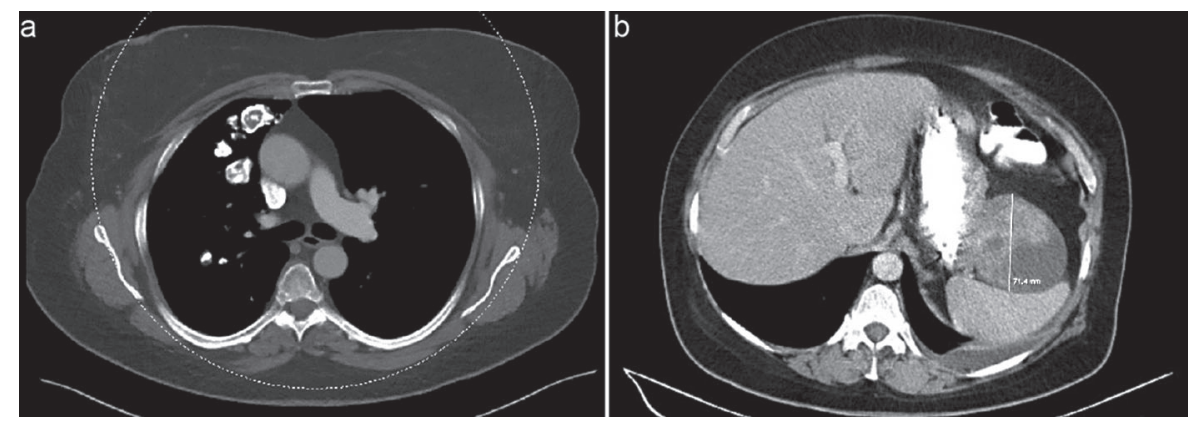

\section{Risk Factors}

There are no known risk factors for GIST. Rarely, they arise in a setting of specific tumor syndromes.

Familial GIST: Only very few reports about a familial GIST can be found in the literature $[3,4]$. In our patients we could not identify a person with a familial GIST. In 1 patient we detected a very rare KIT mutation (exon 8, D418del) that was found in one of the published GIST families although in this family no other GIST tumor has occurred and there are no family ties between our patient and the described family [5].

Carney triad: There is a known association between GIST, pulmonary chondromas, and paragangliomas known as the Carney triad. It is a rare, non-inherited syndrome mainly seen in young females (fig. 1). Carney triad-associated GIST tumors do not normally have a KIT or PDGFRA mutation but show a loss of expression of succinate dehydrogenase subunit B (SDHB) although no mutations of the genes encoding the subunits of SDH are typically identified. The loss of expression is due to alterations in the methylation process of SDH [6-8].

Carney-Stratakis syndrome: In contrast to the Carney triad, this syndrome is a germline mutation of one of the subunits of the SDHA to SDHD with the special risk of developing a GIST and paragangliomas [9].

Type 1 neurofibromatosis (NF Recklinghausen) is an autosomal dominant disorder. Clinically, the patients present with café au lait spots and neurofibromata. The prevalence of the disease is $1 / 3,000$ persons, with half of the patients having a family history. There are several associated conditions including MEN2A or MEN2B syndrome, malignant peripheral nerve sheath tumors, and GIST. The prevalence of GIST in NF1 is estimated at 3.9-25\% mainly in the small intestine, with a median onset of 49 years. Multiple lesions may occur. Typically, there is neither KIT nor PDGFRA mutation nor SDH expression loss [10].

\section{Data Base}

We have more than 800 documented, histologically confirmed GIST patients from 2004 to 2017 at our disposal (fig. 2). Their medium age is 59 years (range: 11-95 years). Only 8/809 patients are younger than 20 years. Men and women are affected with approxi-

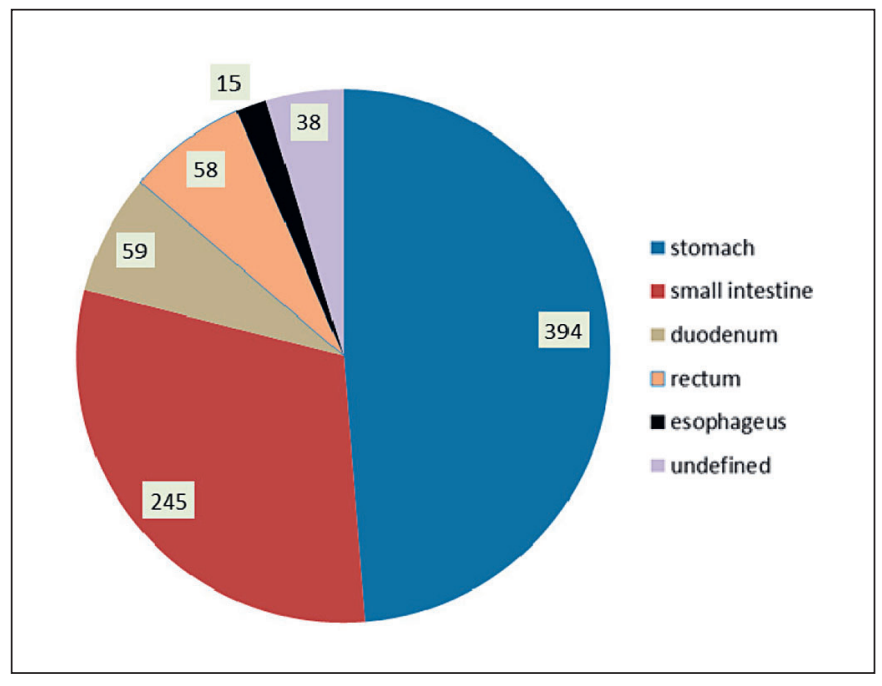

Fig. 2. Location of the primary tumor localization in the Mannheim GIST registry $(n=809)$.

mately similar frequencies, corresponding to 372 females and 432 males. When the registry started to include patients, only the Fletcher and Berman National Institutes of Health classification was available [11], which is still used as a basic documentation. We see a higher proportion of female patients presenting with metastatic disease at initial diagnosis compared to men (fig. 2), but less very low-risk tumors.

\section{Tumor Location and Size}

GISTs can be located in every part of the gastrointestinal tract but may occur in the abdominal cavity as well, including the greater omentum and mesentery. In the literature, Joensuu et al. [12] described the distribution of GIST tumors over the gastrointestinal tract as follows: stomach (60\%), small intestine (30\%), and colon/rectum (5\%); the incidence of esophageal GIST is less than 1\%. [13] This is in accordance with the findings in our GIST population. Among the 391 patients, 192 (59\%) GISTs were located in the stomach, $33(8 \%)$ in the duodenum, $121(30 \%)$ in the small bowel, 30 (8\%) in the rectum, and 5 (1\%) in the esophagus (fig. 2). In 10 patients, the tumor origin could not be defined. However, 
there were another 9 cases initially listed as so-called E-GIST in whom the primary tumor could clearly be detected during later laparotomy. Typically, the first surgery detected peritoneal metastases and the primary tumor was localized in the duodenum or small bowel. Tumor location is an important prognostic factor. In the absence of therapy with tyrosine kinase inhibitors, recurrence in completely resected primary GISTs is independently predicted by mitotic rate, tumor size, and tumor location [14].

The presentation of GISTs is highly variable due to their size and site. In our population, the average tumor size is $75 \mathrm{~mm}$, with a range of $4 \mathrm{~mm}$ to $35 \mathrm{~cm}$. In a population-based study in the preimatinib era, Nilsson et al. [15] found a tumor size of $8.9 \mathrm{~cm}$ in patients with clinical symptoms referring to $70 \%$ of the GIST patients. In contrast, the tumor size was only $2.7 \mathrm{~cm}$ in patients without clinical symptoms (20\%) and $3.4 \mathrm{~cm}$ in patients with GIST detected at autopsy (10\%) [15].

\section{Clinical Presentation of GIST}

In many patients, GIST is an accidental finding, and tumors are often detected with unspecific symptoms. Abdominal diagnostic measures are directed towards mucosal lesions like ulceration or inflammation, whereas pathological conditions of the muscularis layer are typically not expected. If GIST patients present with acute symptoms, the most common clinical presentation is upper gastrointestinal bleeding and gastric discomfort or ulcer-like symptoms. The bleeding varies from chronic insidious bleeding leading to chronic anemia to acute life-threatening episodes of melena or hematemesis (fig. 3). This might also depend on the health system; in this regard, a survey on 150 GIST cases from India showed that due to their transmural growth, a significant percentage of GISTs gain an extraordinarily big size before being diagnosed [16].

There is no pathognomonic feature of GISTs; the symptoms vary a lot according to tumor size and - more importantly - tumor location. A consecutive analysis of 47 patients from Italy found that the most common symptom was abdominal pain and that bleeding in the digestive tract and abdominal pain were more frequent in gastric GISTs (58 and 61\%, respectively), whereas acute abdominal symptoms were more frequent in jejunal and ileal GISTs (40 and 60\%, respectively) [17]. Figure 4 depicts the difference in presentation and diagnosis of the four locations stomach, duodenum, small bowel, and rectum in our database. The classification of symptoms separates patients who had their tumor detected as an emergency (acute upper gastrointestinal bleeding) due to symptoms of the GIST. 'Other acute' means that the GIST was detected during examinations for another acute illness like cholecystitis, tubo-ovarial abscess formation, or abdominal trauma. The term 'latent' describes patients with chronic symptoms (anemia, melena, regurgitation) that could logically be attributed to GIST. 'Screening' means that an abdominal examination was performed as a check-up procedure. The rate of emergency presentation was $9 \%$ for stomach GISTs, $14 \%$ for duodenal GISTs, and $16 \%$ for small bowel GISTs.

Clinical Presentation of Gastrointestinal Stromal Tumors

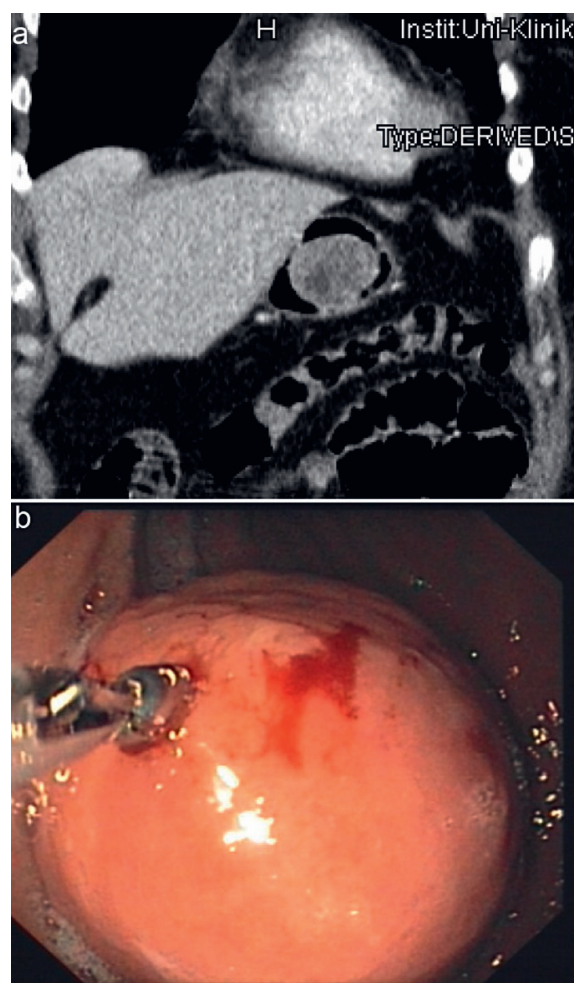

Fig. 3. 67-year-old female symptomatic with acute upper gastrointestinal bleeding from a $6-\mathrm{cm}$ gastric gastrointestinal stromal tumor resected by laparoscopic wedge resection of the stomach after neoadjuvant downstaging with imatinib. a Computed tomography image showing intragastric tumor extension; b endoscopic view and clipping of the superficial bleeding area (endoscopic image courtesy of Prof. Georg Kähler).

\section{Patients Presenting as an Emergency}

In our database, 82 patients presented with acute symptoms and underwent emergency surgery. Of these, 52 patients had a primary tumor localization in the small intestine, 3 patients in the duodenum, and 23 patients in the stomach, whereas in 4 patients the primary tumor could not be localized due to metastatic disease. Interestingly, 11 patients were operated on by gynecologists suspecting torsion of an ovarian cyst or tubo-ovarian abscess. 8 patients underwent laparotomy for suspected Meckel's diverticulum or an acute appendicitis, while a lower or upper gastrointestinal bleeding led to abdominal exploration in 26 patients. In this overall group, 15 patients also presented with a primary ruptured GIST. Looking at the overall group of patients with ruptured GIST, another 11 patients had delayed surgery for a perforated GIST as an elective surgery. In an analysis of GIST-related emergencies from Egypt, Sorour et al. [18] found that among the 92 patients, the most frequent presenting symptom was gastrointestinal bleeding in 45 patients. 26 patients presented with intestinal obstruction, 14 patients with intraperitoneal hemorrhage, and 7 patients with rupture and peritonitis [18].

\section{Clinical Presentation of Gastric GIST}

Gastric GISTs present more frequently with an upper gastrointestinal bleeding (acute hematemesis, melena, or chronic micro- 
Fig. 4. Clinical presentation of gastrointestinal stromal tumor (GIST) depending on the location of the primary tumor, Mannheim GIST registry $(\mathrm{n}=$ 498).

Fig. 5. Unusual location of metastases in gastrointestinal stromal tumor. a Lung metastasis; b bone metastasis; $\mathbf{c}$ retro-ocular metastasis.
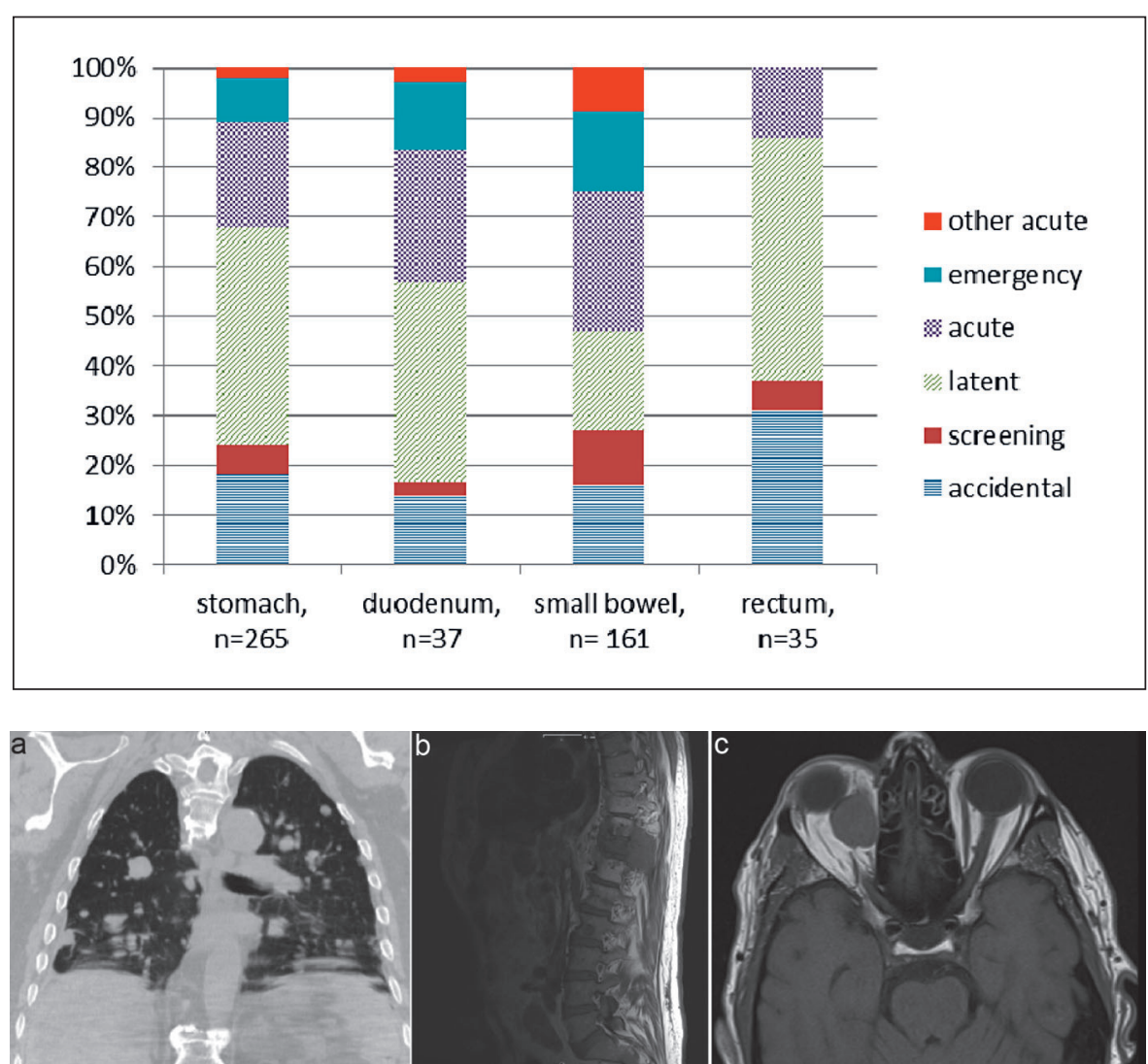

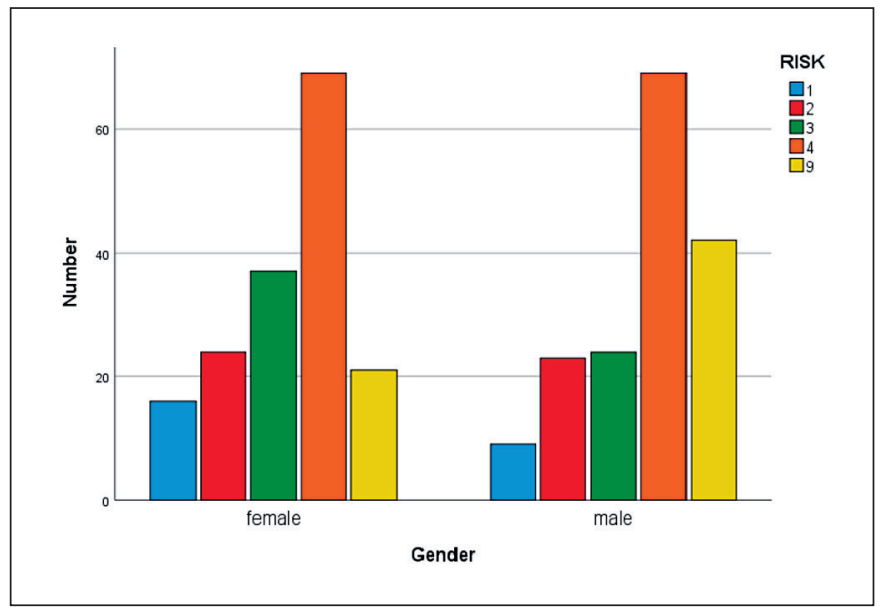

Fig. 6. Comparison of the gender of the patients and their risk classification according to the National Institutes of Health [11]. Differences are statistically significant (Spearman $\mathrm{p}=0.04$, Pearson $\mathrm{p}=0.02) .1=$ Very low risk, $2=$ low risk, $3=$ intermediate risk, $4=$ high risk, $9=$ metastatic disease at initial diagnosis.

cytic anemia) than GISTs in other locations. This bleeding is normally associated with an ulceration of the mucosal surface by the growing submucosal tumor. If visualized in endoscopy, they may be wrongly diagnosed as peptic ulcers if the majority of the tumors grows outside of the stomach. $44 \%$ of our patients presented with latent symptoms.
Clinical Presentation of Small Intestine GIST

Small bowel GISTs are a bit less frequent than stomach GIST but their major emergency presentation is an intestinal obstruction $[17,18]$. In a series from Romania on 30 patients of whom 15 required immediate surgery, Constantin et al. [19] describe the three characteristic conditions for an intestinal obstruction due to an intestinal GIST tumor: obstruction in consequence to the growth of the lesion and direct occlusion of the bowel, intussusception with the tumor as the lead point, or a volvulus-like torsion around the tumor if the tumor growth is extraluminal [19].

\section{Clinical Presentation of Rectal GIST}

Rectal GISTs and extremely few GISTs truly arising in the colon represent only $5 \%$ of all GISTs. There is a male predominance, and the patients either carry a high-risk or low-risk GIST [20] according to the Consensus classification [11]. Clinical presentation of colonic GISTs is less well defined, as tumors are only very rarely found. Gastrointestinal bleeding is the most common clinical presentation of GISTs, but other features may include intestinal obstruction, abdominal pain, perforation, or a palpable pelvic mass, which may be incidentally detected during a gynecological, urological, or endoscopic/radiological procedure or surgery. In our patients, $31 \%$ of GISTs located at the rectum or rectovaginal septum were accidentally detected during digital rectal examination for rectal cancer or during gynecological controls. 50\% of the rectal GIST patients reported latent symptoms of defecation problems, 
Fig. 7. Comparison of survival of our patients compared with their risk classification according to the National Institutes of Health [11]. Note the median survival of 73 months of the cohort presenting with metastatic disease at initial diagnosis. $1=$ Very low risk, 2 = low risk, 3 = intermediate risk, $4=$ high risk, $9=$ metastatic disease at initial diagnosis.

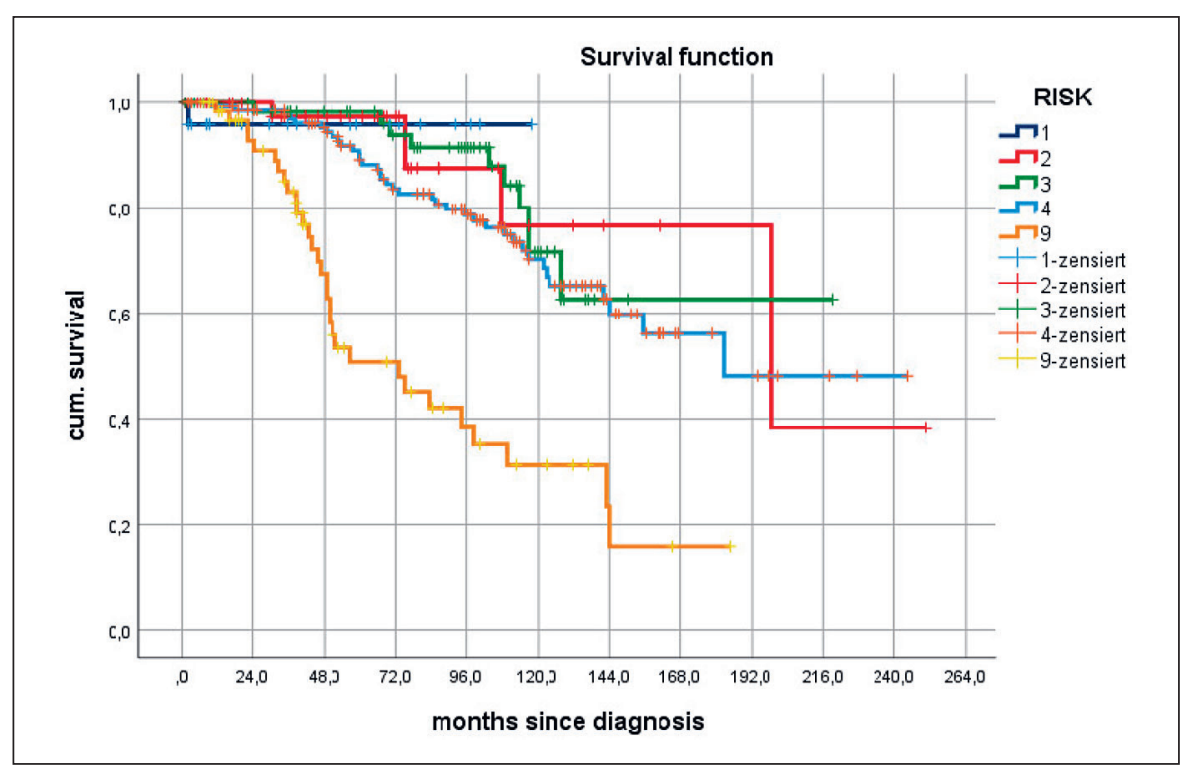

and none of the patients showed up with emergency, acute symptoms (fig. 4).

\section{Micro-/Mini-GISTs}

When GISTs are differentiated by size they are normally classified as micro-GISTs $(<1 \mathrm{~cm})$, mini-GISTs $(1-2 \mathrm{~cm})$, and clinically relevant GISTs $>2 \mathrm{~cm}$. Mini-GISTs are often incidentally found during endoscopy. Micro-GISTs may be mainly identified during postoperative pathologic analysis or during autopsy [21] and have been reported in up to $35 \%$ of patients $>50$ years [22]. Patients with these small GISTs rarely have symptoms and rarely develop disease progression and metastasis despite the fact that these small tumor also harbor mutations in the KIT gene. Yu et al. [23] analyzed 167 patients who underwent endoscopic submucosal dissection for small gastric tumors (size $<3 \mathrm{~cm}$ ) and found a significant amelioration of all components of a dyspepsia symptom score after the intervention in GISTs. Many micro- and mini-GISTs do not progress significantly [24]. In fact, Rossi et al. [25] have suggested that especially micro-GISTs only show a quite low proliferation activity and that features of the tumor cells appear to be benign with low cellularity and sclerosis. In contrast, patients with gastric leiomyomas only partly showed an improvement of their symptoms. The authors concluded that small gastric GISTs may cause dyspeptic symptoms with different pathophysiology in the leiomyoma patients [23].

\section{Are All Submucosal Tumors of the Gastrointestinal Tract GISTs?}

Clearly, not all submucosal tumors thought to present the features of GIST turn out to be GISTs at histological examination of a resection specimen. Differential diagnosis still has to encounter leiomyoma or inflammatory fibroid polyps - which also harbor PDGFRa mutations [26]. Some of the tumors might be recognized from their endoscopic ultrasound features, particularly by increased vascularization to be detected with ultrasound contrast media [27]. The Mannheim data base includes 91 patients with histologically proven GIST excised by laparoscopic resection from the esophagogastric junction, the stomach, and the duodenum. It also lists 35 patients $(27.7 \%, 35 / 126)$ undergoing resection with suspicion of a GIST but later diagnosed as leiomyoma, heterotopic pancreatic tissue, lymphoma, plasmocytoma, or even PEComa. Thus, despite the fact that GISTs are the most common non-epithelial tumors of the gastrointestinal tract, they are not the only group, and diagnosis must be based on pathology.

\section{Pattern of Metastases}

While high-risk GISTs typically metastasize during the first years after diagnosis, GIST metastases have occurred up to 42 years after diagnosis [28]. These data confirm the necessity of long-term follow-up protocols. We analyzed our data base in a subgroup of 731 patients for their metastatic spread with a median follow-up 43.6 months (maximum 274 months). 101 (13.8\%) patients had been initially diagnosed with metastatic disease. Of the remaining 630 patients, 358 patients (56.8\%) developed tumor relapse. Their median time to disease recurrence was 22 months (maximum 144 months), and 302/358 patients (87\%) developed metastases within the abdomen, particularly to the liver $(\mathrm{n}=96)$, the peritoneum $(\mathrm{n}=$ 97), combined liver plus peritoneum $(\mathrm{n}=78)$, and with locoregional recurrence $(n=31)$. In 36 patients, extra-abdominal sites of metastases were found and almost always proven by biopsy: bone $(\mathrm{n}=19)$, soft tissue $(\mathrm{n}=9)$, lung/pleura $(\mathrm{n}=7)$, spleen $(\mathrm{n}=1)$, brain $(n=1)$, ocular $(n=1)$, multiple sites $(n=14)$ (fig. 5$)$. 
Extra-abdominal metastases of GISTs are a rare and late event in the course of disease and occur only after longer therapy with tyrosine kinase inhibitors. Typically, those unusual metastases are observed after the patients have developed liver or peritoneal metastases. They show a rather long median recurrence-free survival after removal of the primary tumor of 58 months (range: 27-105 months). 26 patients had at least two lines of drug therapy prior to developing 'atypical' metastases. These extra-abdominal metastases do not indicate rapid disease progression and may often be controlled by local treatment measures like irradiation if located in the bones or soft tissues.

\section{Impact on Patients Presenting with Metastatic Disease}

Between 10 and $20 \%$ of GIST patients will be diagnosed with overt metastatic disease [12]. As mentioned above, predilection sites for metastases are the liver and peritoneum while metastases outside the abdomen are very rare [29]. In our cohort, there is a statistically significant difference $(\mathrm{p}=0.04)$ between males and females in their stage distribution according to the Consensus [11]. The rate of males presenting with metastatic disease is twice as high as the rate of women (fig. 6). In contrast, with today's molecular characterization of tumors and consecutive treatment with tyrosine kinase inhibitors, patients presenting with metastatic disease have a median survival of 73 months (95\% confidence interval 18108 months) (fig. 7). This is a huge improvement compared with the data reported by DeMatteo et al. [29] from the pre-imatinib era of median 19 months in the year 2000.

\section{Disclosure Statement}

PH declares research support and honoraria from Novartis and AROG. PH and AS received research support through EU FP7 MITIGATE and Novartis. All other authors do not have a conflict of interest to declare.

\section{References}

1 Hirota S, Isozaki K, Moriyama Y, et al: Gain-of-function mutations of c-kit in human gastrointestinal stromal tumors. Science 1998;279:577-580.

2 Nakama A, Hirota S, Okazaki T, et al: Disturbed pyloric motility in Ws/Ws mutant rats due to deficiency of c-kit-expressing interstitial cells of Cajal. Pathol Int 1998;48:843-849.

3 Farag S, van der Kolk LE, van Boven HH, et al: Remarkable effects of imatinib in a family with young onset gastrointestinal stromal tumors and cutaneous hyperpigmentation associated with a germline KITTrp557Arg mutation: case report and literature overview. Fam Cancer 2018;17:247-253

4 Nishida T, Hirota S, Taniguchi M, et al: Familial gastrointestinal stromal tumours with germline mutation of the KIT gene. Nat Genet 1998;19:323-324.

5 Huss S, Kunstlinger H, Wardelmann E, et al: A subset of gastrointestinal stromal tumors previously regarded as wild-type tumors carries somatic activating mutations in KIT exon 8 (p.D419del). Mod Pathol 2013;26: 1004-1012.

6 Killian JK, Kim SY, Miettinen M, et al: Succinate dehydrogenase mutation underlies global epigenomic divergence in gastrointestinal stromal tumor. Cancer Discov 2013;3:648-657.

7 Miettinen M, Killian JK, Wang ZF, et al: Immunohistochemical loss of succinate dehydrogenase subunit A (SDHA) in gastrointestinal stromal tumors (GISTs) signals SDHA germline mutation. Am J Surg Pathol 2013;37:234-240.

8 Carney JA: Gastric stromal sarcoma, pulmonary chondroma, and extra-adrenal paraganglioma (Carney Triad): natural history, adrenocortical component, and possible familial occurrence. Mayo Clin Proc 1999;74: 543-552.

9 Stratakis CA, Carney JA: The triad of paragangliomas, gastric stromal tumours and pulmonary chondromas (Carney triad), and the dyad of paragangliomas and gastric stromal sarcomas (Carney-Stratakis syndrome): molecular genetics and clinical implications. J Intern Med 2009;266:43-52.
10 Agaimy A, Vassos N, Croner RS: Gastrointestinal manifestations of neurofibromatosis type 1 (Recklinghausen's disease): clinicopathological spectrum with pathogenetic considerations. Int J Clin Exp Pathol 2012;5:852-862.

11 Fletcher CD, Berman JJ, Corless C, et al: Diagnosis of gastrointestinal stromal tumors: a consensus approach. Hum Pathol 2002;33:459-465.

12 Joensuu H, Hohenberger P, Corless CL: Gastrointestinal stromal tumour. Lancet 2013;382:973-983.

13 Joensuu H, Vehtari A, Riihimaki J, et al: Risk of recurrence of gastrointestinal stromal tumour after surgery: an analysis of pooled population-based cohorts. Lancet Oncol 2012;13:265-274.

14 Dematteo RP, Gold JS, Saran L, et al: Tumor mitotic rate, size, and location independently predict recurrence after resection of primary gastrointestinal stromal tumor (GIST). Cancer 2008;112:608-615.

15 Nilsson B, Bumming P, Meis-Kindblom JM, et al: Gastrointestinal stromal tumors: the incidence, prevalence, clinical course, and prognostication in the preimatinib mesylate era - a population-based study in western Sweden. Cancer 2005;103:821-829.

16 Yacob M, Inian S, Sudhakar CB: Gastrointestinal stromal tumours: review of 150 cases from a single centre. Indian J Surg 2015;77(suppl 2):505-510.

17 Caterino S, Lorenzon L, Petrucciani N, et al: Gastrointestinal stromal tumors: correlation between symptoms at presentation, tumor location and prognostic factors in 47 consecutive patients. World J Surg Oncol 2011;9:13.

18 Sorour MA, Kassem MI, Ghazal Ael H, El-Riwini MT, Abu Nasr A: Gastrointestinal stromal tumors (GIST) related emergencies. Int J Surg 2014;12:269-280.

19 Constantin VD, Socea B, Popa F, et al: A histopathological and immunohistochemical approach of surgical emergencies of GIST. An interdisciplinary study. Rom J Morphol Embryol 2014;55(suppl):619-627.
20 Jakob J, Mussi C, Ronellenfitsch U, et al: Gastrointestinal stromal tumor of the rectum: results of surgical and multimodality therapy in the era of imatinib. Ann Surg Oncol 2013;20:586-592

21 Nishida T, Goto O, Raut CP, Yahagi N: Diagnostic and treatment strategy for small gastrointestinal stromal tumors. Cancer 2016;122:3110-3118.

22 Foo WC, Liegl-Atzwanger B, Lazar AJ: Pathology of gastrointestinal stromal tumors. Clin Med Insights Pathol 2012;5:23-33.

23 Yu QX, He ZK, Wang J, Sun C, Zhao W, Wang BM: Clinical presentations of gastric small gastrointestinal stromal tumors mimics functional dyspepsia symptoms. World J Gastroenterol 2014;20:11800-11877.

24 Agaimy A, Wunsch PH, Dirnhofer S, Bihl MP, Terracciano LM, Tornillo L: Microscopic gastrointestinal stromal tumors in esophageal and intestinal surgical resection specimens: a clinicopathologic, immunohistochemical, and molecular study of 19 lesions. Am J Surg Pathol 2008;32:867-873.

25 Rossi S, Gasparotto D, Toffolatti L, et al: Molecular and clinicopathologic characterization of gastrointestinal stromal tumors (GISTs) of small size. Am J Surg Pathol 2010;34:1480-1491.

26 Huss S, Wardelmann E, Goltz D, et al: Activating PDGFRA mutations in inflammatory fibroid polyps occur in exons 12, 14 and 18 and are associated with

tumour localization. Histopathology 2012;61:59-68.
27 Yamashita Y, Kato J, Ueda K, et al: Contrast-enhanced endoscopic ultrasonography can predict a higher malignant potential of gastrointestinal stromal tumors by visualizing large newly formed vessels. J Clin Ultrasound 2015;43:89-97.

28 Miettinen M, Lasota J: Gastrointestinal stromal tumors. Gastroenterol Clin North Am 2013;42:399-415.

29 DeMatteo RP, Lewis JJ, Leung D, Mudan SS, Woodruff JM, Brennan MF: Two hundred gastrointestinal stromal tumors: recurrence patterns and prognostic factors for survival. Ann Surg 2000;231:51-58. 